\title{
Ubiquitous Networks: A Need of Future World of Things
}

\author{
Dnyaneshwar S. Mantri*, Pranav M. Pawar, \\ Nandkumar P. Kulkarni and Neeli R. Prasad
}

SIT Lonavala, BITS Pillani Dubai, SKNSCOE Pune, CTIF, Vehicleavatar Inc., USA

E-mail:dsmantri@gmail.com; pranav@dubai.bits-pilani.ac.in;

npkulkarni.pune@gmail.com; neeli.prasad@vehicleavatar.com

${ }^{*}$ Corresponding Author

Received 26 April 2021; Accepted 07 November 2021;

Publication 16 December 2021

\begin{abstract}
With an exponential increase in the number of applications and user demand, it is essential to respond to the query of users with fast services and networks used. This is possible only by the use of ubiquitous networks supporting mass media communications. The integration of advanced technologies such as Communication, Navigation and Sensing Services (CONASENSE) and Human Bond Communications (HBC) takes care of sensing, services, data, speed, cooperation, content, and cost of communication. The combination of Data, Technology, and Media used for intelligent computation and communication over the internet could serve the purpose, and that's the urgent demand of growing networks marching towards a fusion of IoT and $5 \mathrm{G}$ leading to $6 \mathrm{G}$. IoT with $5 \mathrm{G}$ will be the backbone of networks in the future generation network, adding the concept of virtualization at Anytime, Anywhere, Anything, and Anybody. The definition of ubiquitous technology considers it networked, wireless and mobile, to connect a more significant number of users and the world around them. The ubiquitous network connects the D2D, M2M, D2M and uses the ICT and Cloud-based technology to mitigate the
\end{abstract}

Journal of ICT Standardization, Vol. 9_3, 349-370.

doi: 10.13052/jicts2245-800X.933

(C) 2021 River Publishers 
QoS parameters. The paper's primary contribution is the proposal of $6 \mathrm{G}$ enabling technologies and use cases to demonstrate the need and integration of various prime techniques as $\mathrm{IoT}+5 \mathrm{G}+\mathrm{Cloud}+\mathrm{AI} / \mathrm{ML}$. The technology road map and proposed C6-WISDOM model illustrate the fundamentals of enabling future ubiquitous networks $(6 \mathrm{G})$. It also focuses on the critical requirements of $6 \mathrm{G}$ technology in support of ubiquitous networks and identifies the present technologies integrated to provide vertical sustainable wireless networking solutions.

Keywords: Ubiquitous networks, ICT, Cloud computing, Internet of things (IoT), 6G, Convergence and WISDOM.

\section{Introduction}

The current decade is motivated by growing technologies in wireless access networks, machine-machine communication, man-machine interaction, artificial intelligence (AI), and a wide variety of network applications based on it. The emerging area of ubiquitous networks can realize anytime, anywhere communication between object-to-object, person-to-object, and object-object. The use of ubiquitous networks is increasing massively due to the speedy growth of wireless, broadband, and internet technologies [1], supporting everything connected, everywhere, anytime, leading to the digital to virtual to imagination world of things. In all, the role of technological evolution with enabling and supporting techniques is essential. These techniques lead to support for any data formats with increasing data rates. To accommodate and provide solutions with increased data rates, the current and emerging wired and wireless technologies are envisioned the latest development as 6G, which has a major role in the development of wireless communications by taking into consideration the three major aspects as, Mobile ultra-broadband (Terahertz communications), Super Internet-of-Things (IoT) (inter-dependent and Satellite Communications), and Artificial Intelligence (AI) and Machine Learning (ML) [1-3]. The critical network that supports any data rates, latency, and throughput is the Ubiquitous Network. These networks not only support for heterogeneity of networks but also provides the next generation service platforms. The ubiquitous networks are the context-aware, zero-touch networks of computing devices connected at any time and place. They are useful for exchanging information among any devices, networks, people, and objects through the internet and terms as the Internet of Things (IoT). The industrial revolutions replace the term IoT as Internet of Everything (IoE). 
The challenges in the ubiquitous network are Seamless connectivity, Context awareness, Resource management, and Device management [3].

Network connectivity is a significant challenge to know network interface management and seamless connectivity. Context-aware management comprises the adaption, discovery of context, storage, and access control. In the case of resource management, the decimation of information about the machines and devices and resource scheduling in large-scale networks decide which resource is used for the appropriate job. In device management, tracking and location access are essential, along with identification.

The speedy and massive development of wireless, broadband, and internet technologies continuously promoting the use of the ubiquitous network to an advanced level with flexible service platforms to access data from everything connected, everywhere, anytime: Digital to Virtual. According to applications and services, the network has no limits to imagination. The applications used in the ubiquitous networks are SMART and are shown in Figures 1 and 2.

- Precision agriculture

- Environmental monitoring

- Infrastructure management

- Energy management

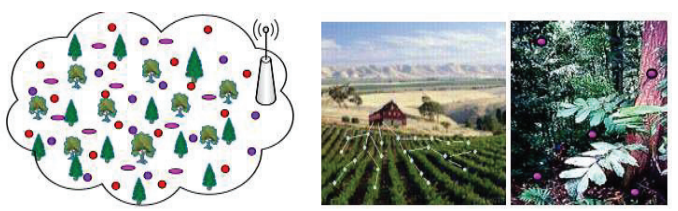

Figure 1 Heterogeneous applications of Ubiquitous networks.

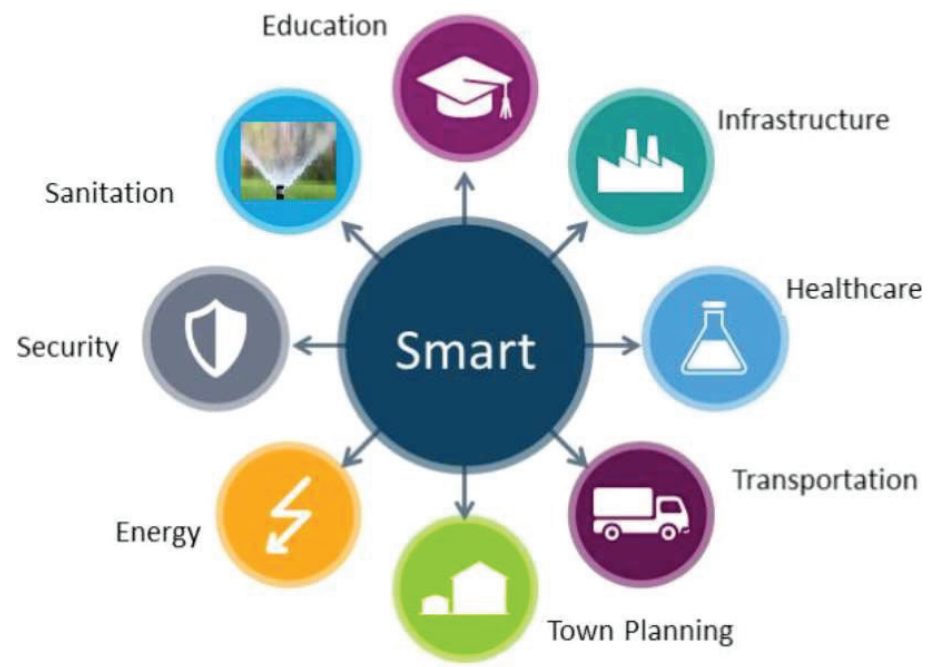

Figure 2 Applications of Ubiquitous networks [smart city]. 
These applications are limited to homogeneous networks and take care of heterogeneous networks (Social to monitoring).

The ultimate goal is to automate human life. The best example of ubiquitous networks application is the smart city, where each element needs to be taken care of by considering the various components as Infrastructure, Health care, Transportation, Planning, Education, Security, Safety, Energy saving, Connectivity, Coordination, and Cooperation [4]. The networks used must have access to the generated data and be utilized for quick services as and when required with less latency and reduced energy utilization. The role of service providers and platforms used is important. The smart city concept illustrated in Figure 1b has four major components: human, technology, data, and Energy. The effective utilization of available resources in smart cities needs management of Energy, Waste, Water, Mobility, Security, transportation, and ICT technology. The involvement of secure wireless connectivity and IoT technology are transforming the traditional elements of city life.

The practical use case of connected car using ubiquitous networks is shown in Figure 3. V2X, a new way of communication between the user, cars, and infrastructure, permits to envision numerous applications focusing on Trusteed Cooperative communication in VANET as heterogeneous communications. The communication is between V2V: Vehicle to Vehicle, V2I: Vehicle to Infrastructure, V2D: Vehicle to Device, V2H: Vehicle to

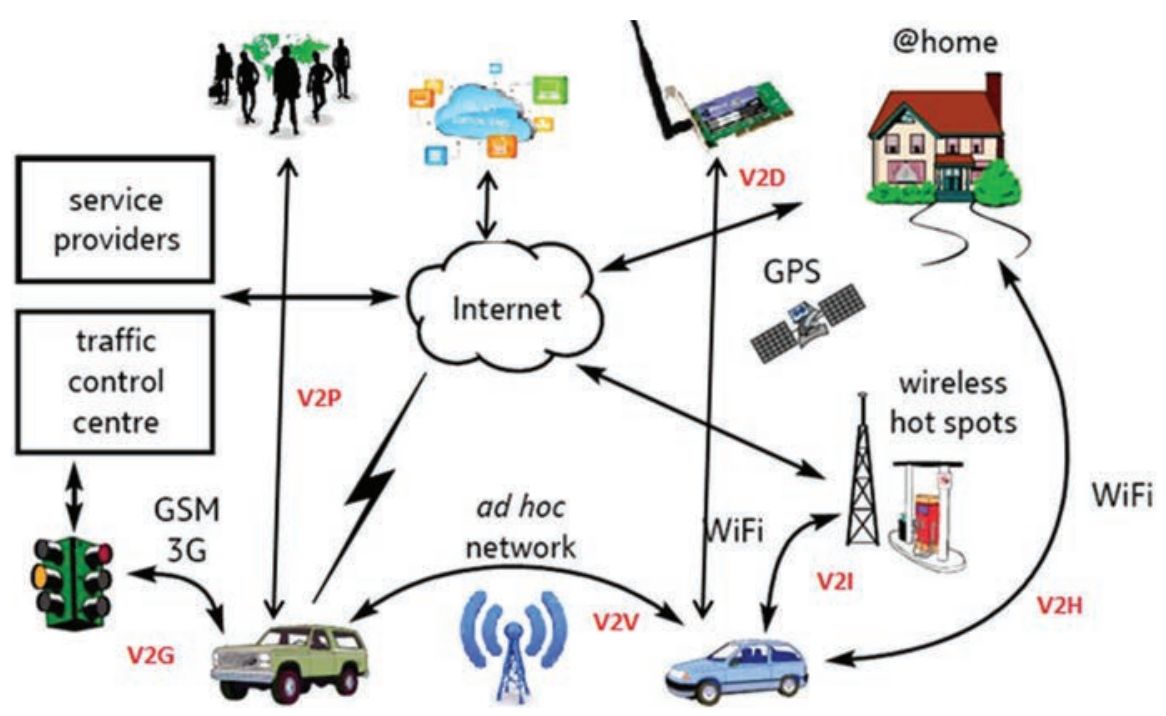

Figure 3 Ubiquitous networks [connected car scenario]. 
House, V2G: Vehicle to Grid, and V2P: Vehicle to Person. V2N: Vehicle to Network. Most of the D2D communication protocols use mobile nodes for extended data delivery. If the system considers the local service, then direct communication between end-users is preferred. During natural disasters like earthquakes, the traditional network does not work due to damage. The need arises to develop an Adhoc network that communicates between D2D, D2M, D2P, and vice versa. With the involvement of IoT, hybrid network will be created for interconnection and communication of accurate data. The best example is shown in Figure 3, V2X communication. The D2D communication in the cellular network uses high data rates and offers the advantage of improved energy efficiency, throughput, latency, and fairness [5, 6]. The different challenges in such applications are Data Traffic Volume, Number of Connected Devices, Diverse Requirements, Energy Consumption, Bandwidth Utilization, Maintaining Privacy and Trust, and Network Connectivity.

The contribution of the paper is to make aware of the latest technological developments and challenges of the fully digital and connected world of things enabling the $6 \mathrm{G}$ ubiquitous networks. The article also focuses on the wave of technological developments of $6 \mathrm{G}$ with integration and convergences of emerging technologies which helps to provide 6G services anytime, anywhere for everything. In continuation with waves of development, the paper introduces the WISDOM (Wireless Innovative System for Dynamic Operations in Mega-communications) model as a base for enabling efficient utilization of $6 \mathrm{G}$ technology.

The paper's significant contribution is the proposal of $6 \mathrm{G}$ enabling technologies and use cases to demonstrate the need and integration of various prime techniques as IoT $+5 \mathrm{G}+\mathrm{Cloud}+\mathrm{AI} / \mathrm{ML}$. The technology road map and proposed C6-WISDOM model illustrate enabling future ubiquitous networks (6G).

To predict the un-predictable things in a network,

- It is necessary to have the vision to plan in the right direction.

- An insight into the future is needed to enable us to take advantage of the present opportunities.

- Support of technology integrations (Ubiquitous Networks)

The broad purpose of this paper is to make aware of the critical requirements of 6G technology in support of ubiquitous networks and identify the present technologies which are integrated to provide the vertical sustainable wireless networking solutions. The paper considers the potential scenarios according to the applications to make human life more comfortable, e.g., 
e-health, smart city, intelligent transportation, D2D and M2M communications with future connected systems. The paper identifies the WISDOM model that supports future networks to go beyond 5G. The authors' analysis suggests that to meet the growing demands of users and networks, it is necessary to have fusion, convergence, and integration of communication technologies, network architectures, and deployment models.

The paper is arranged in different sections starting with Related work (Section 2): which specifies the present work with advantages and disadvantages; Waves of technological development (Section 3): represent the flow of networks from $1 \mathrm{G}$ to $6 \mathrm{G}$ along with various characteristics; Enabling technologies (Section 4): gives the contribution of multiple techniques to meet the objectives of $6 \mathrm{G}$ technology; Road map to new $6 \mathrm{G}$ technology (Section 5): signifies the developments according to industrial revolutions and finally the proposal for $6 \mathrm{G}$ enabler. Conversions, WISDOM model, and Challenges (Section 6): explains the need for Convergence of Technologies and C6 paradigm for the WISDOM model used in 6G and challenges. Finally, the paper is concluded in Section 7.

\section{Related Work}

The section gives the finding and contributions of different authors in the development of $6 \mathrm{G}$ ubiquitous technology. It also gives ideas for integrating various technologies to meet the objectives of $6 \mathrm{G}$ and the future direction for the new proposal.

The work in [6] discusses the concept of V2X communication and the use of AI/ML algorithms for effective communications and data analysis. The paper envisions the various ML techniques used in communication, networking, and security and enables the AI for $6 \mathrm{G}$ vehicular Networks. The research describes in [7] consider the connectivity between People, Vehicle, Sensors, Data, and Cloud resources and updates the system level prospective of $6 \mathrm{G}$ scenarios and requirements according to the evolution of networks from $1 \mathrm{G}$ to $6 \mathrm{G}$. The authors in [8] focus on the details about the D2D, and M2M communications can be effective for localized events using LTE-A; it improves the performance of both communications. The research considered in [9] considers aspects and requirements of rural health solutions according to the quality and available connectivity; without connectivity, the network is useless even though you have technologies like $\mathrm{THz}$ band communication and various AI/ML algorithms for data analysis. Things will play a vital role in pervasive connectivity. The [10] explains the use 
of an AI-based decentralized data model in 6G supporting the internet of everything (IoET). The current application and trend today are switching from IoT-to-IIoT-to-IoET. It also discusses the open issue of envisioned $6 \mathrm{G}$ integrated ubiquitous, intelligent, and decentralized architecture. The [11] discusses the requirements of a $6 \mathrm{G}$ wireless network with a specific focus on the impacts of AI. It also identifies the promising critical technologies for $6 \mathrm{G}$ as $\mathrm{THz}$ communications, Supermassive Multiple -input and Multipleoutput system (SM-MIMO), Holographic Beam Forming (HBF), and Large Intelligent Surfaces (LISs).

The research discussed in [12] addresses the problem of connectivity for people living in rural or underdeveloped areas worldwide. A significant contribution is a detailed survey of technologies for providing ubiquitous connectivity to rural areas, including access/front haul and backhaul techniques. The article also discusses major problems, threats, energy needs, and costeffectiveness of implementing these strategies, with a particular emphasis on the deployment of an electricity network, which is necessary for deploying communication networks. The work in [13] presents IoT based computing framework to present a novel generalized architecture for context-aware ubiquitous learning systems. Four different versions of the proposed architecture were implemented using a standard IoT edge unit. AMQP, CoAP, MQTT, and EMPP (long for each) were used to create the variants; among all the four variants, the performance differences were so minor and gave a choice to choose the variants based on reliability, payload, and protection. The [14] highlights ubiquitous power IoT challenges and recommends an intelligent, collaborative tracking scheme based on advanced parameter prediction skills and enhanced particle filter approaches. The author's recommendation is motivated by edge-cloud interplay's software-defined and function virtualization capabilities. In the first step, the range-based positioning problems are first transformed into a vector nonlinear suboptimal estimation problem using information fusion. Secondly, the density function is used to measure the mobile node's positions and trajectories by collecting cubature points, updating state estimates, and revising vectors. To obtain higher accuracy, the Gauss-Newton iterative method was used. Through experiments, the authors have demonstrated that the proposed algorithm improves efficiency and reliability compared to existing candidates.

The [15] provides a scalable, secure, and effective name service for a ubiquitous futuristic network. The authors proposed SMILE - IoT name services based on five constraints: security, mobility, infrastructure independence, localization, and efficiency after evaluating the requirements. 
The authors scrutinized the DNS's functionality and performance and its current deployment model to see what advantages and disadvantages it has in meeting the SMILE requirements. The article summarizes the numerous DNS-based name services and clean-slate designs that could be used in various application scenarios. The authors from [16] proposed a novel Heterogeneous Distributed Deep Neural Networks (HDDNN) architecture. It provides the intelligent computing environment for cloud, edge (fog), hierarchical distributed end-users in the ubiquitous networks. The article suggests three forms of heterogeneity: (a) node heterogeneity, (b) neural network, and (c) system task. To better serve DNNs, distributed computing architecture is improved. To fully use ubiquitous computing tools, DNNs that are aware of distributed computing systems are developed. The proposed architecture has added benefits of scalability in hardware usage, better accuracy, security, and response time. The research in [17] addresses the 5G and 6G capabilities according to new developments with the added feature of AI/ML. The case study and business model for the convergence of technologies in VANET along with cloud is discussed in [18]. In all, to envision the requirement of $6 \mathrm{G}$ ubiquitous networks, it is essential to have a fusion of multiple technologies (i.e., Convergence and Integration).

\section{Wave of Technological Developments}

Each generation of technology has been designed with added features according to demands from end-users and network operators such as mobile data volumes, number of connected devices, low latency, end-user data rates, battery life for common power devices, reliability, channel bandwidth, and mobility of devices. All these parameters are essential to meet the requirement of 6G networks as a fully Digital and Connected World providing data from flexible service platforms. The wave of technological developments to meet the need of 6G technology is shown in Figure 4.

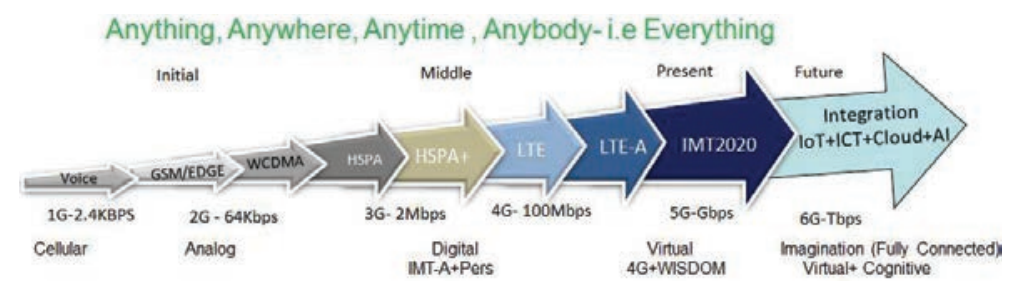

Figure 4 Wave of technology developments (toward 6G). 
Table 1 Comparison of evolution of Technologies $1 \mathrm{G}$ to $6 \mathrm{G}[1-3,10,11]$

\begin{tabular}{|c|c|c|c|c|c|c|}
\hline $\begin{array}{l}\text { Parameter/ } \\
\text { Evolution }\end{array}$ & $1 G$ & $2 \mathrm{G}$ & $3 \mathrm{G}$ & $4 \mathrm{G}$ & $5 \mathrm{G}$ & $6 \mathrm{G}$ \\
\hline Year & 1980 & 1990 & 2000 & 2010 & 2020 & $2025-30$ \\
\hline Data Rates & $2.4 \mathrm{Kbps}$ & $64 \mathrm{Kbps}$ & $2 \mathrm{Mbps}$ & $\begin{array}{l}100-1000 \\
\text { Mbps }\end{array}$ & 1-10 Gbps & 3 Tbps \\
\hline $\begin{array}{l}\text { Standard } \\
\text { Technol- } \\
\text { ogy }\end{array}$ & $\begin{array}{l}\text { AMPS/ } \\
\text { IMPS }\end{array}$ & GSM & WCDMA & $\begin{array}{l}\text { LTE-A, } \\
\text { Wimax }\end{array}$ & $\begin{array}{l}\text { MIMO, } \\
\text { mm-Wave } \\
\text { IMT2020 }\end{array}$ & $\begin{array}{l}\mathrm{THz} \text { commu- } \\
\text { nication } \\
\text { SM-MIMO }\end{array}$ \\
\hline Latency & $1-10$ secs & $\begin{array}{l}300-1000 \\
\mathrm{~ms}\end{array}$ & $\begin{array}{l}100-500 \\
\mathrm{~ms}\end{array}$ & $36-48 \mathrm{~ms}$ & $5 \mathrm{~ms}$ & $1 \mathrm{~ms}$ \\
\hline Frequency & $30 \mathrm{KHz}$ & $1.8 \mathrm{GHz}$ & $1.6-2 \mathrm{GHz}$ & $2-8 \mathrm{GHz}$ & 3-30 GHz & $\begin{array}{l}95 \mathrm{GHz}-3 \\
\mathrm{THz}\end{array}$ \\
\hline $\begin{array}{l}\text { Access } \\
\text { System }\end{array}$ & FDMA & $\begin{array}{l}\text { TDMA/ } \\
\text { CDMA }\end{array}$ & CDMA & CDMA & $\begin{array}{l}\text { OFDM/ } \\
\text { BDMA }\end{array}$ & Conversion \\
\hline $\begin{array}{l}\text { Core } \\
\text { Network }\end{array}$ & PSTN & PSTN & $\begin{array}{l}\text { Circuit } \\
\text { switching }\end{array}$ & $\begin{array}{l}\text { Evolved } \\
\text { Packet Core } \\
{[\mathrm{EPC}]}\end{array}$ & Stand Alone & $\begin{array}{l}\text { Deep, } \\
\text { Intelligent } \\
\text { Integration }\end{array}$ \\
\hline $\begin{array}{l}\text { Internet } \\
\text { services }\end{array}$ & $\begin{array}{l}\text { Narrow } \\
\text { band }\end{array}$ & $\begin{array}{l}\text { Narrow } \\
\text { band }\end{array}$ & Broadband & $\begin{array}{l}\text { Ultra broad } \\
\text { band }\end{array}$ & $\begin{array}{l}\text { Wireless } \\
\text { world wide } \\
\text { web }\end{array}$ & Wireless \\
\hline Application & $\begin{array}{l}\text { Voice } \\
\text { calling } \\
\text { (Land- } \\
\text { lines) }\end{array}$ & Text SMS & $\begin{array}{l}\text { Text SMS, } \\
\text { Internet }\end{array}$ & $\begin{array}{l}\text { Internet }+ \\
\text { Application } \\
\text { (Video) }\end{array}$ & $\begin{array}{l}\text { IoT+ICT, } \\
\text { (Smart } \\
\text { Devices) }\end{array}$ & $\begin{array}{l}\text { Fully Digital } \\
\text { Connected } \\
\text { world } \\
\text { (Location } \\
\text { Awareness) }\end{array}$ \\
\hline Advantages & $\begin{array}{l}\text { Voice } \\
\text { Calls }\end{array}$ & $\begin{array}{l}\text { Suitable } \\
\text { for SMS } \\
\text { and MMS }\end{array}$ & $\begin{array}{l}\text { Provides } \\
\text { security } \\
\text { and } \\
\text { roaming }\end{array}$ & $\begin{array}{l}\text { Data rates are } \\
\text { high with } \\
\text { mobility }\end{array}$ & $\begin{array}{l}\text { Speed of } \\
\text { operation is } \\
\text { high, less } \\
\text { delay }\end{array}$ & $\begin{array}{l}\text { High Energy } \\
\text { efficiency, } \\
\text { low latency }\end{array}$ \\
\hline
\end{tabular}

The targeted goal of wireless networks is to offer better quality, consistent, and secure communication like wired networks. Each new generation of wireless network services represents a big step in the targeted goal of moving from $1 \mathrm{G}$ to $6 \mathrm{G}$. Table 1 shows the comparison of evolution of technologies with respect to parameters year, data rate, standard technology, latency, frequency used, access system, core network, internet services, application and advantage.

\section{Ubiquitous Networks: 6G Enabling Technologies}

As the ubiquitous networks have a variety of homogenous and heterogeneous networks connected for communication and use different protocols for 
communication, they need the exact and intellect interactions between the devices since

- The increase amount video streaming, leading to the rapid increase of mobile data traffic.

- Every user carrying multiple devices, increasing the number of connections.

- Need of IoT network which can handle billions and more devices.

- Energy efficiency needs to grow, with an increased amount of data traffic on the network.

- User wants more services in less tariff, which increases network operators' pressure to reduce operational expenditures.

- The new avenues of increasing revenue are opening for network operators with new use cases and applications due to mobile communication technology advancements.

- While providing services to billions of devices, its most important to maintain authenticity, privacy, and security for trustworthy applications.

Figure 5 depicts the combination of various enabling technologies that make $6 \mathrm{G}$ intelligent. It uses the fusion of different technologies for storage, analysis, and communication in a network with low latency and high throughput. The supportive functions of each enabling technology will be the potential candidate for integration.

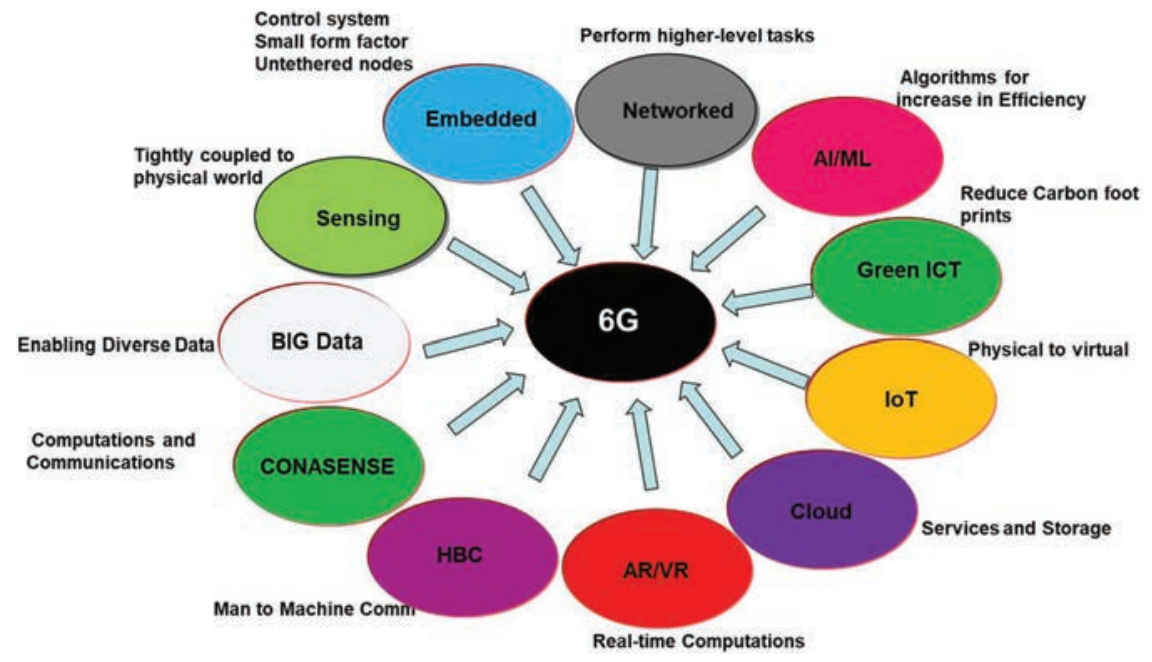

Figure 5 6G Network Enabling Technologies [4-6, 9]. 
The fusion of various technologies such as Artificial Intelligence, Computer Vision, D2D and M2M Communication, Deep Learning, Big Data Analytics, Machine Learning, Green ICT, Cloud Computing, etc., makes 6G ubiquitous technology suitable for high-end applications.

\section{Proposal for 6G Enhancements}

The future world of Things is considered a fusion of multiple techniques used in complementary developments, and a single technology could not serve a purpose. It bridges the gap between the physical, virtual, and imagination worlds (HBC). The 6G technology is the integration and convergence of multiple technologies towards for fully digital and connected world. The capabilities of the ubiquitous networks used in $6 \mathrm{G}$ includes and are not only limited to,

- Cooperative and communication technology

- Network addressing and identification

- Sensing and actuation

- Processing of information at hardware and software level

- Network localization

- User interfaces and auto updates

The road map to enhance the user industry interface revolution and functionality reaching 6G technology is shown in Figure 6. In the modernized digital world, what the end-user expect is a fully digital and connected world of things.

A ubiquitous smart network uses different electronic sensors to collect data and integrates and communicates using ICT platforms. These platforms are helpful for optimizing the efficiency of services for IoT-enabled devices that directly connect with people. According to the concept and requirement to have good governance, the composition for ubiquitous smart networks has components related to Data, Technology, and Communication is shown in Figure 7.

The conversions and integration of smart technologies as Internet of Things, Information Communication Technology and Cloud are the base for $5 \mathrm{G}$ i.e

$$
S M A R T \approx 5 G \approx I o T+I C T+\text { Cloud }
$$

5G has supported providing the data at high rates (Gbps) and does not have communication bandwidth limitations. The infrastructure utilized is flexible to accommodate various applications such as e-Health, smart city, 


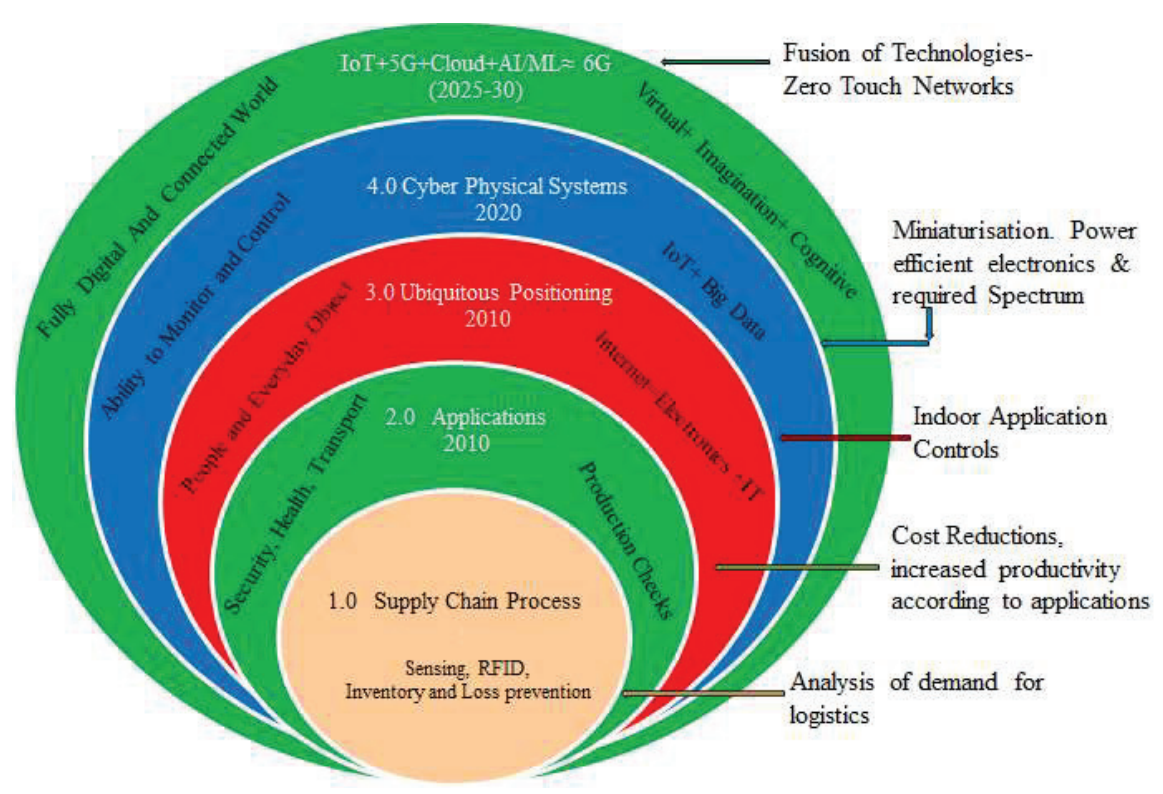

Figure 6 Roadmap to $6 \mathrm{G}$.

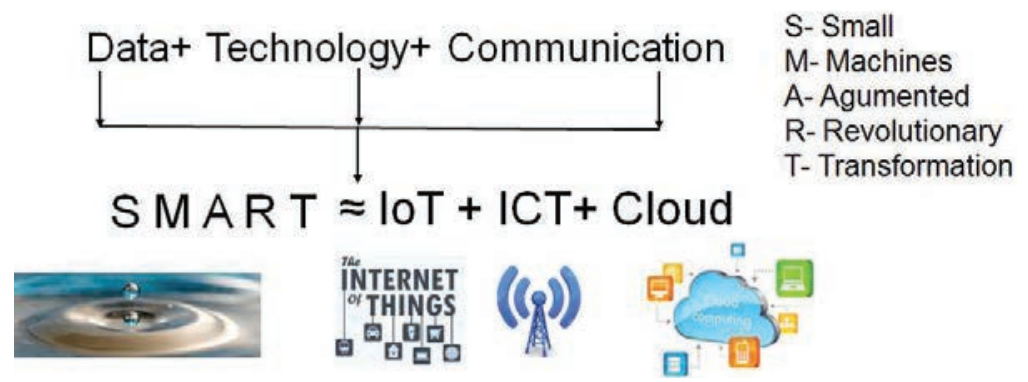

Figure 7 Smart ubiquitous network components.

and public safety in D2D, M2M interfaces with LTE-A. It helps to reduce the end-to-end delay with increased spectral efficiency and the ability to store data virtually $[8,9]$. With the added feature of data analysis in the $5 \mathrm{G}$ and increasing broad spectrum of applications according to Figure 5, the $6 \mathrm{G}$ could have a combination of multiple technologies converged together and can be expressed as,

$$
6 G \approx \text { Virtual }+ \text { Imagivation }+ \text { Cognitive } \approx I o T+5 G+\text { Cloud }+A I
$$


- Multi GBPS Data Rates

- Extreme Capacity

- Uniformity

- Deep Awareness

- Ultra low Latency

- High Reliability

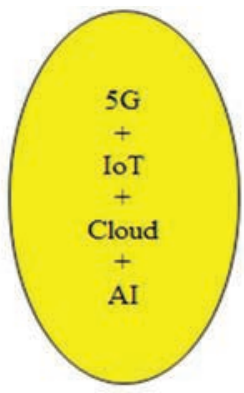

- High Availability

- Strong Security

- Low Cost

- Ultra Low Energy usage

- Deep Coverage

- High density

Figure 8 Expectations of $6 \mathrm{G}$.

After deployment of 5G network around the world, the next generation network $6 \mathrm{G}$ with very low latency and Artificial Intelligence (AI) enabled network will be proposed for future evolutions. The $6 \mathrm{G}$ will impact the development of intelligence communication, which comprises different connectivity aspects as intelligent, deep, holographic, and ubiquitous [3, 17]. The expectations of the proposed 6G network are given in Figure 8.

\section{Convergence, Wisdom Model and Challenges}

In a cooperative environment, it is essential to consider the involvement of research, innovation, and invention with education for leading the development of new emerging technologies. Convergence is an important aspect established for the development of ubiquitous networks [18]. It considers the togetherness of Audio, Video, Fax, Still pictures, Images, decentralized networks, Data fusion techniques that work on accepted data and communicates using wired or wireless medium. Convergence is a synonym to any Technological Evolution.

The ubiquitous networks used in the technological developments towards $6 \mathrm{G}$ must have convergence and integration of,

a. Networks: Wired, Wireless and Broadcast, Ad hoc and Sensor Networks

b. Technology: Computer, Communication, Consumer, IoT, Cloud and $\mathrm{AI} / \mathrm{ML}$

c. Applications: Speech, Data, Graphics, Video, Multimedia and Big data

d. Services: Cloud based, Navigation and communication.

These applications and services lead to the Wireless Innovative System for Dynamic Operations in Mega-communications (WISDOM). It incorporates the $\mathrm{C} 6$ model completing the expected requirements of $6 \mathrm{G}$ networks 


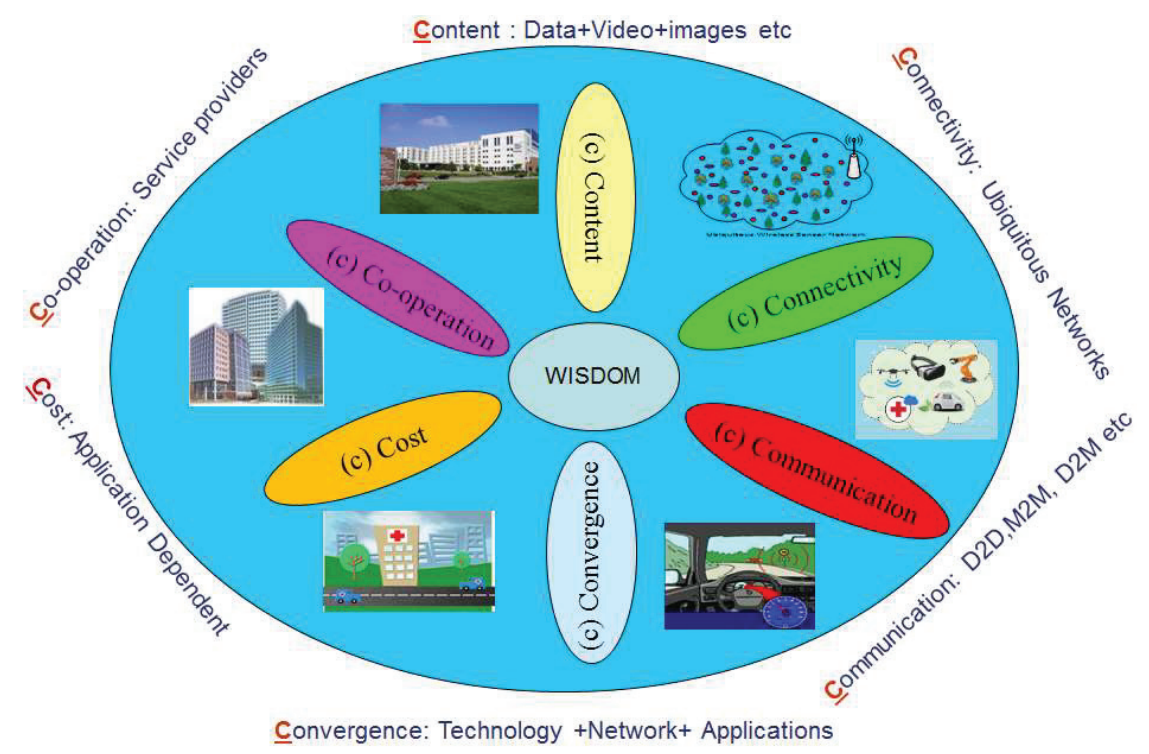

Figure 9 C6-WISDOM Model used in Future Networks (6G).

as added Spectrum, Jumbled technologies, cell-less future networks, Imaginations, Virtualization, Spectral efficiency, Less Latency, More connected Things, computational intelligence, and cooperative communication. The self-explanatory WISDOM model is as shown in Figure 9.

The C6-Wisdom model has various arms as

- Convergence: First most important thing in every high-speed network. It is essential to have a common platform to share the information obtained from the various networks may be wired, or wireless. IoT, Cloud, and ICT with 5G technologies play an essential role since they will be used for control, storage, and communication with low latency. The convergence of Technology, Network, and Application scenarios leads to effective throughput.

- Connectivity: Devices, things, sensors in the network may be in the vicinity of each other or maybe far away, it is essential to have a network, storage, access mechanism, and power availability, in absence of these network connectivity is impossible and without it is impossible to control, monitor and communicate the network application. The most important is to have connectivity and availability of ubiquitous networks with essential resources. 
- Communication: The elements of a network such as devices, machines, people, and things should be able to communicate in a unique data format. The main advantage of a ubiquitous network is that it supports communication with any data format - which increases efficiency. The need is to communicate the data at reduced latency of the order of 0.1 nano-sec. To achieve it communication bandwidth requirement is much higher and must be satisfied. It can be achieved using different techniques such as aggregation, scheduling, and synchronization.

- Contents: With billions of devices connected, data generated may be in the form of Images, Audio, Videos, text, etc. it is of utmost importance to handle and convert into the required format so that it can be used for analysis and control actions.

- Cooperation: The increased number of devices in the network, ultimately data generation rate goes high, but at the same time, if each member of the network starts sending data randomly, the data from users may get collide or may be lost in a network and purpose could not be served. To make sure the received contents are correct, the network must be trained, scheduled, and synchronized cooperatively. This demands cooperation and communication from the network operators and service provides.

- Cost: The most important is cost and depends on the type of application and network used.

\section{Challenges}

The scholars, community observers, and cynics have worries about the capacities of the ubiquitous computing revolution considering development in required infrastructure for enabling $6 \mathrm{G}$ ubiquitous networks:

1. Infrastructure: Flexibility, Cost Effective, Support

2. Data Virtualization: enhancement of service platforms for e.g. Cloud storage

3. Communication: Availability of Network, Connectivity, Low power consumption

4. Spectrum sharing: Need to match data rates (Scheduling and Synchronization)

5. Security and Privacy: Current booming issue in each network

6. Service automation: Virtualization at scale, Closed Loop Assurance, Towards zero touch networks 
7. Device Management: Intra network communication

8. Resource Management: System capacity

9. Maintaining low latency and reliability

10. Increased Energy efficiency: Reduced cost of connectivity and communication

\section{Conclusions}

At an exponential pace, things, machines, and devices connected to the Internet have increased and continue to expand. The Ubiquitous computing technology perspective covers several problems in software and hardware. Critical issues are mobility, scalability, heterogeneity, privacy and trust, reliability, security, bandwidth utilization, scheduling, synchronization, and integration. The significant themes explained in the paper will help in the evolution of 6G Ubiquitous computing (Intelligence, Cloud-based Computing, and Sensor Networks). The WISDOM model proposed is the zero-touch network covering all the requirements of $6 \mathrm{G}$. The best enablers of $6 \mathrm{G}$ are convergence and integration of multiple technologies used in various types of communications not limited to devices or things but also supported with people and industrial revolutions that are changing from IoT-to-IIoT to IoET and match the objective of $6 \mathrm{G}$. The paper uses different terminologies and methods interesting for future studies. Technology is a word that describes something that doesn't work yet - Imagination.

\section{References}

[1] Mohammed H. Alsharif, Anabi Hilary Kelechi,, Mahmoud A. Albreem, "Sixth Generation (6G) Wireless Networks: Vision, Research Activities, Challenges and Potential Solutions," Symmetry 2020, 12, 676; doi:10.3 390/sym12040676.

[2] Ian F. Akyildiz, (Fellow, IEEE), Ahan Kak, and Shuai Nie, "6G and Beyond: The Future of Wireless Communications Systems", IEEE Access, Vol. 8, 2020. pp. 133995-134030.

[3] Yang Lua,, Xianrong Zheng, "6G: A survey on technologies, scenarios, challenges, and the related issues", Elsevier Journal of Industrial Information Integration 19 (2020) 100158.

[4] S. Mohapatra, S. Mohanty, and S. Mohanty, "Smart healthcare: An approach for ubiquitous healthcare management using IoT," in Big 
Data Analytics for Intelligent Healthcare Management. Amsterdam, The Netherlands: Elsevier, 2019, pp. 175-196.

[5] Study on LTE Support for Vehicle-to-Everything (V2X) Services, document TR 22.885, 3GPP , v14.0.0, 2015. Accessed: May 2019. [Online]. Available: http://www.3gpp.org/DynaReport/22885.htm

[6] Fengxiao Tang, Yuichi Kawamoto, Nei Kato, and Jiajia Liu, "Future Intelligent and Secure Vehicular Network Toward 6G: MachineLearning Approaches", Proceedings of the IEEE, Vol. 108, No. 2, pp. 292-307, February 2020. doi: 10.1109/JPROC.2019.2954595.

[7] Marco Giordani, Michele Polese, Marco Mezzavilla, Sundeep Rangan, Michele Zorzi, "Towards 6G Networks: Use Cases and Technologies", arXiv:1903.12216v2 [cs.NI] 4 Feb. 2020.

[8] B. Raghothaman, "Architecture and Protocols for LTE based device to device communications", Inc. Proc. ICNC 2013, pp. 895-899.

[9] Jedari, Behrouz; Xia, Feng; Ning, Zhaolong, "A Survey on HumanCentric Communications in Non-Cooperative Wireless Relay Networks", IEEE Communi. Surveys \& Tutorials. Vol. 20, No. 2, pp. 914 944, 2018, arXiv:2008.04651 doi:10.1109/COMST.2018.2791428.

[10] Xiuquan Qiao, Schahram Dustdar, Yakun Huang, Junliang Chen, "6G Vision: An AI-Driven Decentralized Network and Service Architecture", Department: Internet of Things, People, and Processes, IEEE Computer Society, pp. 33-40, Sept. 2020. doi:10.1109/MIC.2020.2 987738.

[11] Z. Zhang, Y. Xiao, Z. Ma, Ming Xiao, Z. Ding, X. Lei, G.K. Karagiannidis and P. Fan, "6G Wireless Networks, Vision, Requirements, Architecture, and Key Technologies", IEEE Vehicular, Technology Magazin, pp. 28-41, Sept. 2019, doi:10.1109/MVT.2019.2921208.

[12] Elias Yaacoub, and Mohamad Salim Alouini,"A Key 6G Challenge and Opportunity-Connecting the Base of the Pyramid: A Survey on Rural Connectivity”, Proceedings of IEEE, Vol. 108, No. 4, April 2020. pp. 533-582 doi:10.1109/JPROC.2020.2976703.

[13] Salsabeel Y. Shapsough, and Imran A. Zualkernan, Member, IEEE, "A Generic IoT Architecture for Ubiquitous Context-Aware Learning", IEEE TRANS. On Learning Methodologies, Vol. 13, No. 3, pp. 449464, July-Sept. 2020. doi:10.1109/TLT.2020.3007708.

[14] Fei Song, Mingqiang Zhu, Yutong Zhou, Ilsun You, and Hongke Zhang, "Smart Collaborative Tracking for Ubiquitous Power IoT in Edge-Cloud Interplay Domain", IEEE, Internet of Thingd Journal, Vol. 7, No. 7, pp. 6044-6055, July 2020, doi:10.1109/JIOT.2019.2958097. 
[15] Zhiwei Yan, Hongtao Li, Sherali Zeadally, Yu Zengi, and Guanggang Geng, "Is DNS Ready for Ubiquitous Internet of Things?", IEEE Access, Vol. 7, pp. 28835-28846, 2019. doi:10.1109/ACCESS.2019. 2901801.

[16] Zongpu Zhang, Tao Song, Liwei Lin, Yang Hua, Xufeng He, Zhengui Xue, Ruhui Ma, and Haibing Guan, "Towards Ubiquitous Intelligent Computing: Heterogeneous Distributed Deep Neural Networks", IEEE Transaction on BIG DATA, pp. 01-15, doi:10.1109/TBDATA.2018.28 80978.

[17] Anutusha Dogra, Rakesh Kumar Jha, and Shubha Jain, "A Survey on beyond 5G network with the advent of 6G: Architecture and Emerging Technologies", IEEE Access, pp. 1-37, doi:10.1109/ACCESS.2020.30 31234.

[18] Nandkumar Kulkarni, Neeli Rashmi Prasad, Tao Lin, Mahbubul Alam, Ramjee Prasad, "Convergence of Secure Vehicular Ad-Hoc Network and Cloud in Internet of Things", River Publications, Role of ICT for Multi-Disciplinary Applications in 2030, Book Chapter, ISBN: 9788793379480, 2016.

\section{Biographies}

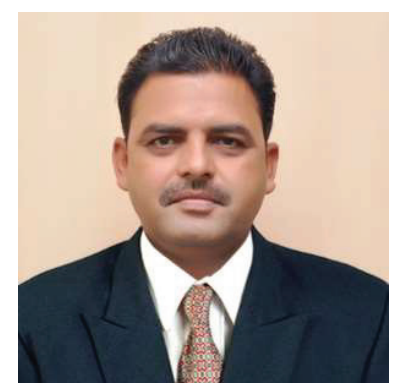

Dnyaneshwar S. Mantri is graduated in Electronics Engineering from Walchand Institute of Technology, Solapur (MS) India in 1992 and received Masters from Shivaji University in 2006. He has awarded Ph.D. in Wireless Communication at Center for TeleInFrastruktur (CTIF), Aalborg University, Denmark. He has teaching experience of 25 years. From 1993 to 2006 he was working as a lecturer in different institutes [MCE Nilanga, MGM Nanded, and STB College of Engg. Tuljapur (MS) India]. 
From 2006 he is associated with Sinhgad Institute of Technology, Lonavala, Pune and presently he is working as Professor in Department of Electronics and Telecommunication Engineering.

$\mathrm{He}$ is IEEE member, Life Member of ISTE and IETE. He has published three books, 09 Journal papers in indexed and reputed Journals (Springer, Elsevier, IEEE etc.) and 19 papers in IEEE conferences. He is reviewer of international journals (Wireless Personal Communication, Springer, Elsevier, IEEE, Communication society, MDPI etc.) and conferences organized by IEEE. He worked as TPC member for various IEEE conferences and also organized IEEE conference GCWCN2014. He worked on various committees at University and College. His research interests are in Adhoc Networks, Wireless Sensor Networks, Wireless Communications specific focus on energy and bandwidth.

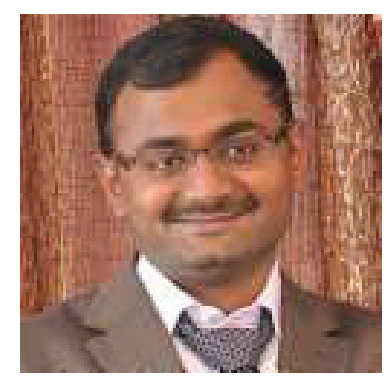

Pranav M. Pawar graduated in Computer Engineering from Dr. Babasaheb Ambedkar Technological University, Maharashtra, India, in 2005, received Master in Computer Engineering from Pune University, in 2007 and received $\mathrm{PhD}$ in Wireless Communication from Aalborg University, Denmark in 2016, his $\mathrm{PhD}$ thesis received nomination for Best Thesis Award from Aalborg University, Denmark. Currently he is working as an Assistant Professor in Dept of Computer Science, Birla Institute of Technology and Science, Dubai, before to BITS he was a postdoctoral fellow at Bar-Ilan University, Israel during March 2019 to Ocotober 2020 in an area of Wireless Communication and Deep Leaning.

$\mathrm{He}$ is recipient of outstanding postdoctoral fellowship from Israel Planning and Budgeting Committee. He worked as an Associate Professor at MIT ADT Univerisy, Pune from 2018-2019 and also as an Associate Professor in Department of Information Technology, STES's Smt. Kashibai Navale College of Engineering, Pune from 2008-2018. From 2006 to 2007, was working 
as System Executive in POS-IPC, Pune, India. He received Recognition from Infosys Technologies Ltd. for contribution in Campus Connect Program and also received different funding for research and attending conferences at international level. He published more than 40 papers at national and international level. He is IBM DB2 and IBM RAD certified professional and completed NPTEL certification in different subjects. His research interests are Energy efficient MAC for WSN, QoS in WSN, wireless security, green technology, computer architecture, database management system and bioinformatics.

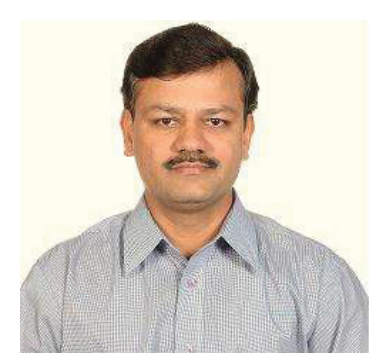

Nandkumar P. Kulkarni received Bachelor of Engineering (B.E.) degree from Walchand College of Engineering, Sangli, Maharashtra, (India) in 1996. He has been with Electronica, Pune from 1996-2000. He worked on retrofits, CNC machines and was also responsible for PLC programming. In 2000, he received the Diploma in Advanced Computing (C-DAC) degree from MET's IIT, Mumbai. In 2002, he became Microsoft Certified Solution Developer (MCSD). He worked as a software developer and system analyst in CITIL, Pune and INTREX India, Mumbai respectively. He has 23 years of experience both in industry and academia. From 2002 onwards he worked as a faculty in Savitribai Phule Pune University, Pune. Since 2007, he is working with SKNCOE, Pune as a faculty in IT Department. He completed a Master of Technology (M. Tech) degree with computer specialization from COEP, Pune (India) in 2007 and Ph.D. from Aarhus University, Denmark in 2019. His area of research is in WSN, VANET, and Cloud Computing. He has published papers in 18 International Journals, 15 International IEEE conferences, 03 National Conferences. 


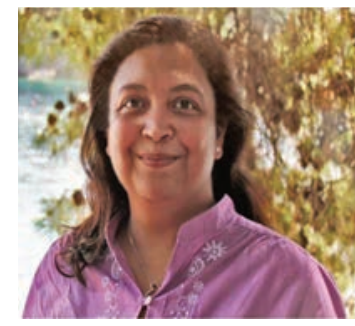

Neeli R. Prasad, CTO of SmartAvatar B.V. Netherlands and VehicleAvatar Inc. USA, IEEE VTS Board of Governor Elected Member \& VP Membership. She is also full Professor at Department of Business Development and Technology (BTech), Aarhus University. Neeli is a cybersecurity, networking and IoT strategist. She has throughout her career been driving business and technology innovation, from incubation to prototyping to validation and is currently an entrepreneur and consultant in Silicon Valley.

She has made her way up the "waves of secure communication technology by contributing to the most ground breaking and commercial inventions. She has general management, leadership and technology skills, having worked for service providers and technology companies in various key leadership roles. She is the advisory board member for the European Commission H2020 projects. She is also a vice chair and patronage chair of IEEE Communication Society Globecom/ICC Management \& Strategy Committee (COMSOC GIMS) and Chair of the Marketing, Strategy and IEEE Staff Liaison Group.

Dr. Neeli Prasad has led global teams of researchers across multiple technical areas and projects in Japan, India, throughout Europe and USA. She has been involved in numerous research and development projects. She also led multiple EU projects such as CRUISE, LIFE 2.0, ASPIRE, etc. as project coordinator and PI. She has played key roles from concept to implementation to standardization. Her strong commitment to operational excellence, innovative approach to business and technological problems and aptitude for partnering cross-functionally across the industry have reshaped and elevated her role as project coordinator making her a preferred partner in multinational and European Commission project consortiums. 
She has 4 books on IoT and WiFi, many book chapters, peer-reviewed international journal papers and over 200 international conference papers. Dr. Prasad received her Master's degree in electrical and electronics engineering from Netherland's renowned Delft University of Technology, with a focus on personal mobile and radar communications. She was awarded her Ph.D. degree from Università di Roma "Tor Vergata", Italy, on Adaptive Security for Wireless Heterogeneous Networks. 